doi: $\underline{10.31211 / \text { interacoes.n39.2019.a5 }}$

\title{
Impacto social de la política de legalización de Cannabis sativa (marihuana) en Uruguay
}

\author{
Ignacio Pablo Traversa Tejero \\ SNI. Sistema Nacional de Investigadores, Uruguay - igtraversa@gmail.com
}

\begin{abstract}
Resumen
La Marihuana (Cannabis sativa L.) es una planta con fines múltiples, el psicoactivo es el más diversificado y globalizado. En Uruguay esta droga fue legalizada, el objetivo de ésta investigación fue conocer el impacto social de su legalización. Fueron aplicados cuestionarios en seis municipios. Se encontró que la droga provoca efectos múltiples, un tercio de los uruguayos la probó al menos una vez, los canales de acceso a la droga son

informales, aunque las vías legales alcanzan un tercio del total. Las tres cuartas partes de la población desconoce el objetivo de la ley, por lo que su valoración cuantitativa en escala 1-10 es poco favorable $(4,3)$. Además, piensan que conduce al consumo de otras drogas y que afecta el trabajo, el estudio y la vida social. El consumo es independiente del nivel económico. La población cree que la ley atrae al turismo cannábico.
\end{abstract}

Palabras clave: Cannabis, política pública, impacto social, psicoactivo.

\section{Impacto social da política de legalização da Cannabis sativa (marijuana) no Uruguai}

\begin{abstract}
Sumário
A marijuana (Cannabis sativa L.) é uma planta com múltiplos usos, a psicoativa é a mais diversificada e globalizada. No Uruguai esta droga foi legalizada. O objetivo deste estudo foi identificar o impacto social da sua legalização. Foram aplicados questionários em seis municípios. Verificou-se que a droga causa múltiplos efeitos, um terço dos uruguaios já a utilizaram pelo menos uma vez, e que os canais de acesso ao medicamento são

informais, embora os meios legais cheguem a um terço do total dos inquiridos. Três quartos da população desconhecem o objetivo da lei, portanto a sua avaliação quantitativa na escala 1-10 é desfavorável $(4,3)$. Além disso, consideram que a legalização leva ao consumo de outras drogas e afeta o trabalho, o estudo e a vida social. O consumo é independente do nível económico. A população inquirida acredita que a lei atrai o turismo de cannabis.
\end{abstract}

Palavras-chave: Cannabis, política pública, impacto social, psicoativo. 


\title{
Social impact of legalization of Cannabis sativa (marijuana) in Uruguay
}

\begin{abstract}
Marijuana (Cannabis sativa L.) is a multipurpose plant, the psychoactive is the most diversified and globalized. In Uruguay this drug was legalized, the objective of this investigation was to know the impact of its legalization. Questionnaires were applied in six municipalities. It was found that the drug causes multiple effects, a third of Uruguayans tested it at least once, the access channels to reach a third of the total. Three-quarters of the population does not know the purpose of the law, so its quantitative assessment on a 1-10 scale is unfavorable (4.3). In addition, they think that it leads to the consumption of other drugs and that it affects work, study and social life. The consumption is independent of the economic. Half of the interviewees believe that the law attracts cannabis tourism.
\end{abstract}

Keywords: Cannabis, public politics, social impact, psychoactive.

\section{INTRODUCCIÓN}

Hace más de 4000 años, la Marihuana (Cannabis sativa L.) ha sido utilizada con múltiples fines, tales como: médicos, recreativos y religiosos, a partir de fibras textiles, usos medicinales, propósitos recreativos, en la gastronomía, y en lienzos para pinturas. Sus hojas presentan resina y contienen cerca de cuatrocientos compuestos químicos de los cuales sesenta pertenecen al grupo de los cannabinoides (Rodríguez et al., 2005). Esta planta posee propiedades psicoactivas y es considerada una de las drogas más consumidas en el mundo (UNODC, 2012)

En diferentes países, algunos de los factores que favorecen el consumo de drogas ilícitas son: el carácter competitivo de la actual sociedad, la incomunicación social, la discriminación, el abandono, la desorganización de la estructura familiar, los antecedentes familiares de consumo de alcohol y la existencia de redes de narcotráfico en gran parte del mundo (Quiroga et al., 2008).

En el Uruguay, ciertos sectores sociales se movilizaron por la legalización del cannabis en 2007 cuando se unieron tres organizaciones constitutivas: Laplacita, Prolegal y Plantatuplanta (Filardo et al., 2012); en tiempos en que los sectores políticos ya 
proponían su legalización como una medida dirigida desde la óptica de seguridad y protección hacia los derechos de los usuarios (Repetto, 2014). A partir del debate sobre la elaboración y implementación de esta ley se empezó a construir en la sociedad uruguaya un escenario con menos prejuicios, creando nuevas narrativas que posibilitaron un análisis más crítico y profundo y abierto a nuevas alternativas de políticas flexibilizadoras (de Armas Bernardi, 2016), sobretodo, considerando el fin medicinal popularizado que jugó a favor de la legalización (León Cam, 2017).

En marzo de 2011 en Uruguay se introdujo una proyecto de ley que luego no prosperó, en este se proponía permitir el porte de 25 gr. de marihuana, con la autorización de sembrar ocho plantitas (Filardo et al., 2012). A la fecha mencionada Uruguay es el único país de la región que ha aprobado la legalización de la marihuana (Rodríguez Florez, 2013), con la permisión del cultivo de seis plantas y un máximo de 480 gramos de recolección anual (Ley 19. 172-2014, 2014).

Luego del alcohol y del tabaco, la marihuana es la droga más consumida en Uruguay, para acceder a ella los consumidores debían delinquir al entrar en el mercado de narcotráfico (Rovira et al., 2014). De acuerdo con Repetto (2014) con la Ley Nº. 19.172 del año 2014 se pretendería interferir en los negocios de los narcotraficantes, teniendo como resultado adicional un aumento en los ingresos fiscales para el estado, además de permitir que las fábricas médicas, farmacéuticas y demás productoras de sustancias lícitas puedan hacer uso de cannabis para la creación de nuevos estupefacientes. Por su parte, Arocena y Aguiar (2017) mencionan que el poder ejecutivo de Uruguay apostó a que la regulación de la producción de C. sativa pueda ser una forma eficaz de combatir parte del narcotráfico y de esta manera contribuir a disminuir el crimen y la violencia.

Bajo la hipótesis de trabajo de falta de información y desconocimiento por parte de los ciudadanos o una posible polarización sobre el tema, los objetivos implicados en esta investigación están enfocados a conocer el impacto de la legalización de ésta droga a la vez de identificar la posible existencia del turismo cannábico sobre todo en la zona norte del país donde existe una frontera "seca" con el Brasil de fácil tránsito para el turista. De acuerdo con el Índice de desarrollo humano (IDH) la zona fronteriza tiene a Uruguay con un valor de 0,765 (escala 0-1) ocupando la posición 52 del ranking y a Brasil $(0,699)$ ocupando la posición 73 (PNUD, 2010a). 


\section{METODOLOGÍA}

\section{Área de estudio}

El área muestreada se correspondió con los departamentos fronterizos del norte del Uruguay con Brasil. Esta frontera es más virtual que real dado que se permite un tránsito facilitado de personas y un fácil acceso de los turistas, es por ello que fueron analizados los departamentos uruguayos de Artigas, Rivera, Cerro Largo Tacuarembó y los municipios brasileros de Santana do Livramento y Quaraí. La faja fronteriza se extiende en la dirección sureste-noroeste. Se trata de una región que concentra la población en las ciudades, debido a ello la toma de datos se realizó en las capitales municipales. Solamente el departamento de Rivera concentra un $31 \%$ del total de la población de los cuatro departamentos uruguayos fronterizos (Mazzei \& de Souza, 2013). Los departamentos uruguayos analizados fueron: Artigas, Rivera, Tacuarembó y Cerro Largo (Figura 1).

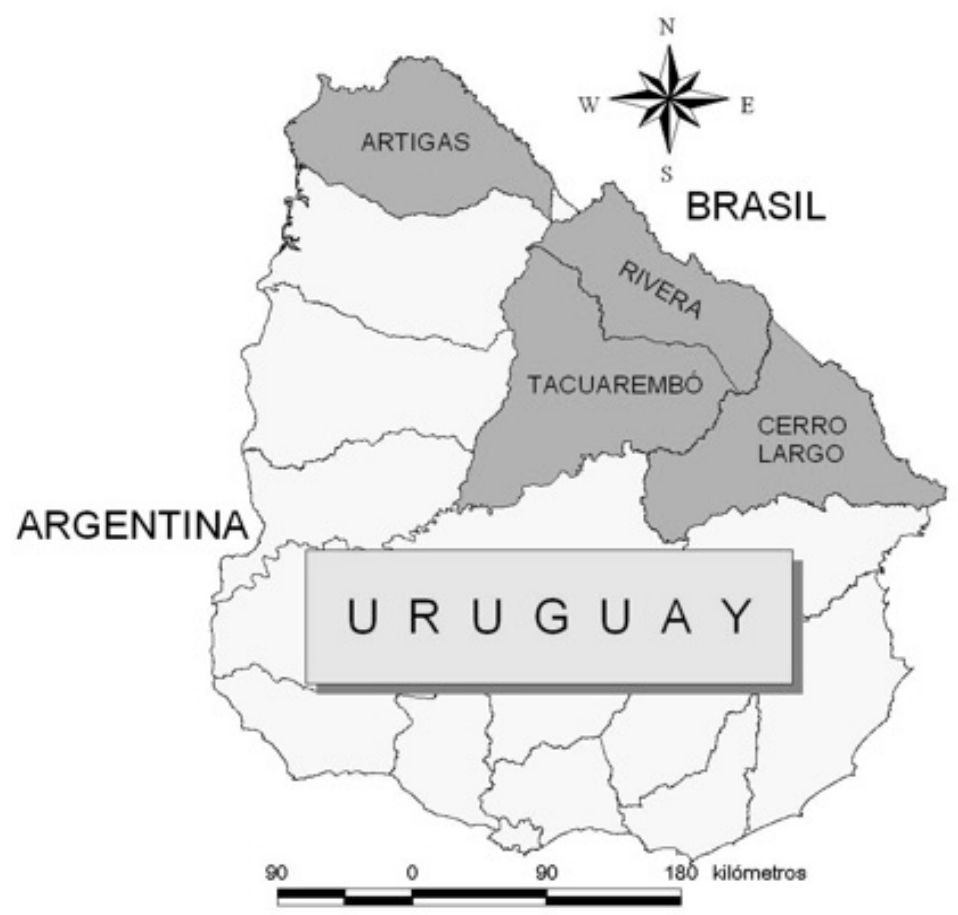

Figura 1. Área relevada (departamentos) de Uruguay. 
En los últimos años ha ocurrido un leve descenso de la población fronteriza uruguaya, de acuerdo con la serie histórica la distribución poblacional fue de 326.333 personas (1996) y 329.657 personas (2011); por su parte, en la frontera brasilera ocurrió un fenómeno semejante, el censo del año 2000 estimó un total de 481.899 personas y el censo del año 2010, 468.821 habitantes (Mazzei \& de Souza, 2013). El índice de desarrollo humano (valores 0-1) regional es muy similar para todos los municipios estudiados: Artigas 0,727, Rivera 0,739, Cerro Largo 0,720, Tacuarembó 0,736 (OTU, 2010), Quarai 0,704, Livramento 0,727 (PNUD, 2010b).

\section{Recolección de datos}

Se definieron espacios públicos como aquellas zonas con acceso no limitado, de uso compartido, que son oportunidades de encuentro, de aparición del otro, vinculadas al habitar común (Filardo et al., 2012). En estos espacios se aplicaron cuestionarios mixtos (preguntas abiertas y cerradas) (Baranger, 2006). Se diseñó y aplicó una encuesta (Valdés Vento et al., 2009), a manera de entrevista informal consistente en una conversación oral entre dos personas (entrevistado y entrevistador) (Roesch, 2009; Marconi \& Lakatos, 2011). Las preguntas cerradas fueron tipificadas para facilitar la codificación y su posterior procesamiento (Baranger, 2006).

El tamaño de la muestra (n) fue seleccionado teniendo en cuenta la distribución normal estándar:

$$
n=\frac{\left(Z_{\alpha}\right)^{2} * p(1-p)}{m^{2}}
$$

donde " $Z_{\alpha}$ " $(1,96)$ fue el nivel de confianza para un $\alpha=0,05$ (confianza 95\%), "p" $(0,05)$ fue la probabilidad estimada $(5 \%)$; "m" el margen de error admisible de 5\%, (valor estandarizado 0,05) (Infante \& Zárate de Lara, 1994). De acuerdo con la formula anterior, el valor de $\mathrm{n}$ es igual a 73 unidades de muestreo para cada departamento (municipio) estudiado. Para los seis municipios estudiados fueron necesarias 438 unidades de muestreo. A los efectos de cubrir con más seguridad el margen de error los entrevistados fueron 440 personas). Fueron ocho variables estudiadas en los encuestados: si se declara consumidor de marihuana, si conoce los efectos a la hora de consumirla, su postura frente a la legalización de la Marihuana, si tiene conocimiento sobre la razón de la implementación da la ley, si manifiesta interés en la 
marihuana, los usos que se le podría dar a la marihuana, la valoración del impacto de la legalización en Uruguay y las consecuencias sobre la Marihuana. De manera global, se consideró impacto social como la sumatoria de las percepciones de los sujetos frente a los aspectos considerados en los cuestionarios que fueron aplicados durante el período de julio a noviembre de 2018, derivados de la implementación de la ley 19172, promulgada por el Poder Ejecutivo en diciembre de 2013 y reglamentada en mayo de 2014.

\section{Procesamiento de Datos}

Los datos recabados fueron procesados por medio de funciones estadísticas de la planilla de cálculo Microsoft Excel, en donde se aplicaron métodos de análisis numéricos (parámetros de tendencia central y de dispersión), métodos tabulares y métodos gráficos (Baranger, 2009). Los resultados de los cuestionarios se agruparon por frecuencias absolutas (fi) y por frecuencias relativas (hi), está última calculada como fi sobre el número total de observaciones (n). Se consideró a la frecuencia relativa como un buen estimador de la probabilidad teórica buscada cuando el tamaño de la muestra es grande (Spiegel et al., 2014).

Las respuestas a las preguntas abiertas del cuestionario (no tipificadas, ej. efectos del consumo) fueron categorizadas por afinidad o similitud de las afirmaciones para poder así estimar las frecuencias relativas (probabilidad).

\section{RESULTADOS Y DISCUSIÓN}

Las drogas estimulantes producen: aumento de la atención, estado de alerta, actividad psicomotora y del sistema nervioso autónomo, actúan sobre mecanismos neurológicos produciendo placer conductual, usurpan los mecanismos dopaminérgicos pero no producen efecto de saciedad, es decir que el aumento de la dopamina no se detiene (mecanismo básico de la adicción) (Mazzei, 2018). En esta investigación fue hallado que quienes son consumidores manifestaron efectos muy variados (Tabla 1). 


\section{Tabla 1}

Efectos de C. sativa por frecuencias

\begin{tabular}{|c|c|c|}
\hline Efecto & $\begin{array}{c}\text { Frecuencia } \\
\text { relativa }\end{array}$ & $\begin{array}{l}\text { Frecuencia } \\
\text { relativa }(\%)\end{array}$ \\
\hline Relajación-paz & 0,196 & 19,6 \\
\hline Alegría-Felicidad-Risas & 0,121 & 12,1 \\
\hline Euforia-Excitación-Adrenalina & 0,112 & 11,2 \\
\hline Sueño & 0,112 & 11,2 \\
\hline Hambre & 0,089 & 8,9 \\
\hline Tranquilidad & 0,075 & 7,5 \\
\hline Alucinaciones y Delirio & 0,037 & 3,7 \\
\hline Bienestar-placer & 0,028 & 2,8 \\
\hline Desorientación-Descoordinación-Desconcentración & 0,023 & 2,3 \\
\hline Mareos & 0,023 & 2,3 \\
\hline Alivio-Calmante & 0,023 & 2,3 \\
\hline Hipersensibilidad & 0,019 & 1,9 \\
\hline Sed & 0,019 & 1,9 \\
\hline Aburrimiento-Pereza & 0,014 & 1,4 \\
\hline Sedante & 0,014 & 1,4 \\
\hline Taquicardia-Presión alta & 0,014 & 1,4 \\
\hline Vómitos & 0,014 & 1,4 \\
\hline Ansiedad & 0,009 & 0,9 \\
\hline Ojos rojos & 0,009 & 0,9 \\
\hline Pánico & 0,009 & 0,9 \\
\hline Reanimación & 0,009 & 0,9 \\
\hline Tristeza-Depresión & 0,009 & 0,9 \\
\hline Calor & 0,005 & 0,5 \\
\hline Disminución de conciencia & 0,005 & 0,5 \\
\hline Insomnio & 0,005 & 0,5 \\
\hline Violencia & 0,005 & 0,5 \\
\hline Total & 1,000 & 100,0 \\
\hline
\end{tabular}


Los hallazgos concuerdan con Ruiz y Próspero (2014) quienes afirman que la marihuana provoca una serie de cambios en múltiples sistemas neuroquímicos y deteriora las funciones cognitivas al provocar que el cerebro procese más lentamente la información que percibe del medio ambiente. De acuerdo con Escobar Toledo et al. (2009) serían los cannabinoides los causales, dado que tienen efectos duales de agresividad y apatía.

La población fronteriza estudiada revela un apreciable nivel de consumo de $C$. sativa, aproximadamente un tercio de los entrevistados han consumido alguna vez o regularmente (Tabla 2).

Tabla 2

Efectos de C. sativa por frecuencias.

\begin{tabular}{lc}
\hline \multicolumn{1}{c}{ Nivel de consumo } & frecuencia relativa $\%$ \\
\hline nunca consumieron & 68,1 \\
alguna vez consumieron & 22,4 \\
regularmente consumen & 9,5 \\
Total & 100,0 \\
\hline
\end{tabular}

A nivel mundial el nivel de consumo se mantuvo relativamente bajo, no obstante, en los años sesenta, estudios demostraron un aumento drástico del consumo de marihuana con fines recreativos, principalmente en adolescentes y adultos jóvenes (Rodríguez, 2012). En el año 2006, la marihuana era la droga ilegal más consumida en Uruguay, no obstante sólo el 12,2\% de la población declaraba haber experimentado esta droga alguna vez en su vida, con un consumo mayor en Montevideo (16,7 \%) contra apenas el 6,6 \% en interior del país que es el área que se corresponde con esta investigación (ONU, 2006).

La extensión del consumo a nivel mundial tiene vínculos con la creación de canales o vías de adquisición del producto; en Uruguay todavía se encuentran niveles altos de canales informales de adquisición, donde el mercado negro constituye aún el $40 \%$, aunque no son despreciables los modos formales de compra en farmacias y de autocultivo quienes sumados totalizan un tercio del total (Tabla 3). 
Tabla 3

Modos de adquisición de C. sativa.

\begin{tabular}{lcc}
\hline \multicolumn{1}{c}{ Modo de adquisición } & $\begin{array}{c}\text { Frecuencia } \\
\text { relativa \% }\end{array}$ & $\begin{array}{c}\text { Frecuencia } \\
\text { absoluta }\end{array}$ \\
\hline Mercado negro- boca de droga & 39,5 & 85 \\
amigos-conocidos-vecinos & 21,9 & 47 \\
farmacias & 17,2 & 37 \\
autocultivo-plantación & 15,3 & 33 \\
plazas-bailes & 2,8 & 6 \\
Total & 96,7 & 208 \\
\hline
\end{tabular}

Cuestionados los encuestados sobre los objetivos de la implementación de la legalización de C. sativa, aproximadamente las tres cuartas partes manifestaron no saber el porqué y el $20 \%$ para combatir el narcotráfico (Tabla 4 ).

Tabla 4

Objetivos de la Ley de legalización de C. sativa.

\begin{tabular}{rc}
\hline Objetivo de la Ley & Frecuencia relativa \% \\
\hline combate narcotráfico & 77,40 \\
control compra-venta & 18,08 \\
ganancias al estado & 2,17 \\
controlar consumidores & 0,54 \\
permitir venta en farmacias & 0,54 \\
reducir el consumo & 0,36 \\
aumentar la seguridad & 0,18 \\
abaratar el producto & 0,18 \\
facilitarle al consumidor & 0,18 \\
obtención de votos & 0,18 \\
Total & 0,18 \\
\hline
\end{tabular}

Una asociación recurrente unifica droga y delincuencia, esta transitiva aparece en todo el espacio social y con particular intensidad en las posiciones económicas inferiores y en las personas mayores, en la medida que disminuye la edad aparecen matices, distinciones, fundamentalmente entre la marihuana y la pasta base (Filardo et al., 2012). 
En este estudio se encontró una postura mayoritaria poco favorable ante la ley, dado que el $73 \%$ de los encuestados no tiene interés sobre el tema frente a una minoría de $27 \%$ que si lo tiene. En cuanto a la postura frente a la legalización $36 \%$ dijo estar de acuerdo, $46 \%$ en desacuerdo y el resto no saber y en relación a la valoración del impacto de la ley, el reporte más frecuente fue regular (40\% aproximadamente), no obstante la valoración mala y muy mala sumadas totalizan otro $40 \%$ (Figura 2). En escala numérica (1-10) la valoración tiene un promedio de 4,3 un desvío estándar 6,1.

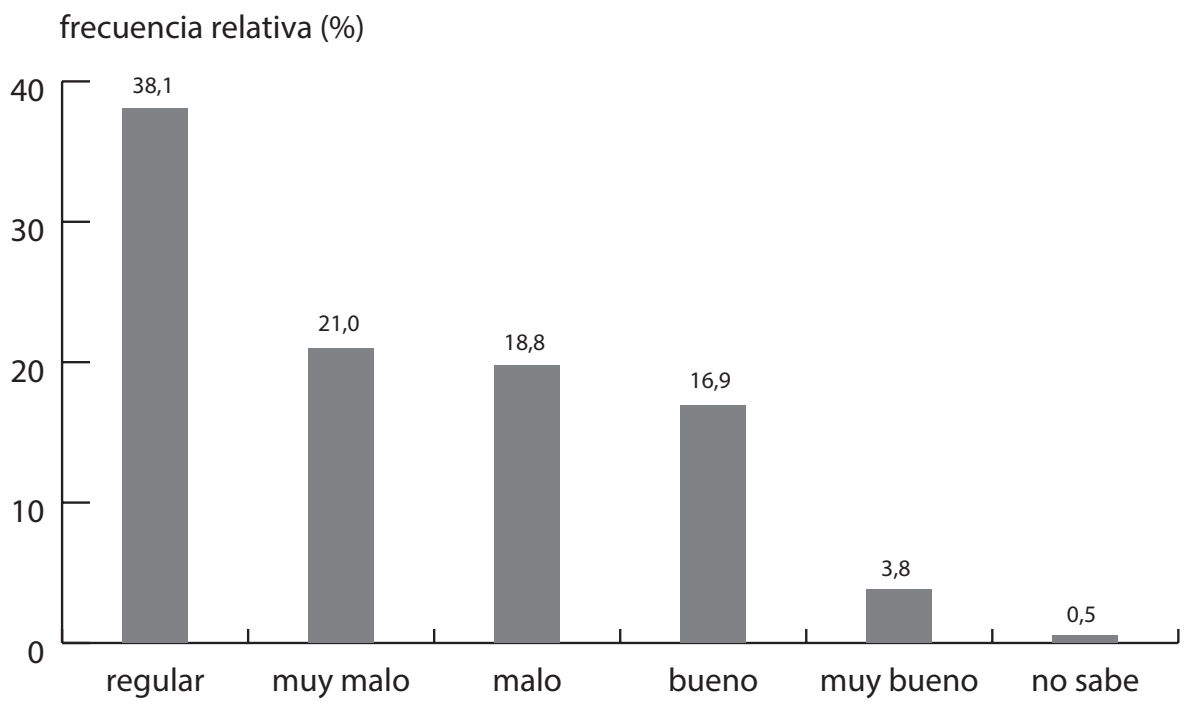

Figura 2. Valoración del impacto de la legalización de C. sativa.

En Montevideo, la percepción de un aumento del consumo y sus consecuencias públicas se extiende en todo el espacio social, pero la interpretación dada a sus consecuencias y la retórica fundante varían en relación con la edad, por ejemplo entre los más mayores predomina una visión más lejana, más abstracta de la droga, que equipara a las distintas sustancias ilegales en un conjunto, y que las asocia rápidamente con la delincuencia, también creciente (Filardo et al., 2012). Es posible que esta valoración negativa general esté basada en la creencia de que C. sativa induce al consumo de otras drogas (73\%). De los entrevistados 287 del total manifestaron el vínculo con una amplia diversidad de drogas (Tabla 5) y (Figura 3). 
Tabla 5

Vínculo de C. sativa con otras drogas.

\begin{tabular}{rcc}
\hline Inducción a otras drogas & Frecuencia absoluta & Frecuencia relativa \% \\
\hline cocaína & 106 & 36,9 \\
pasta base & 44 & 15,3 \\
crack & 43 & 15,0 \\
alcohol & 27 & 9,4 \\
todas & 25 & 8,7 \\
éxtasis & 11 & 3,8 \\
tabaco & 10 & 3,5 \\
lsd & 14 & 4,9 \\
heroína & 4 & 1,4 \\
metanfetaminas & 2 & 0,7 \\
opio & 1 & 0,3 \\
Total & 287 & 100,0 \\
\hline
\end{tabular}

La droga extraída de C. sativa es la más consumida en el mundo, mantiene un ritmo de crecimiento sostenido y una disminución en la edad de inicio. En las últimas décadas la percepción de riesgo asociada al consumo entre estudiantes de secundaria, descendió de 60\% en 1994 a 37\% en 2004, lo cual da la pauta de una relación entre alto consumo y baja percepción o noción de riesgo (CONACE, 2005). Además, hay estudios que muestran que existe una relación entre el consumo de marihuana y el rendimiento académico (Gorosteguy \& Viani, 2009). 
frecuencia relativa (\%)

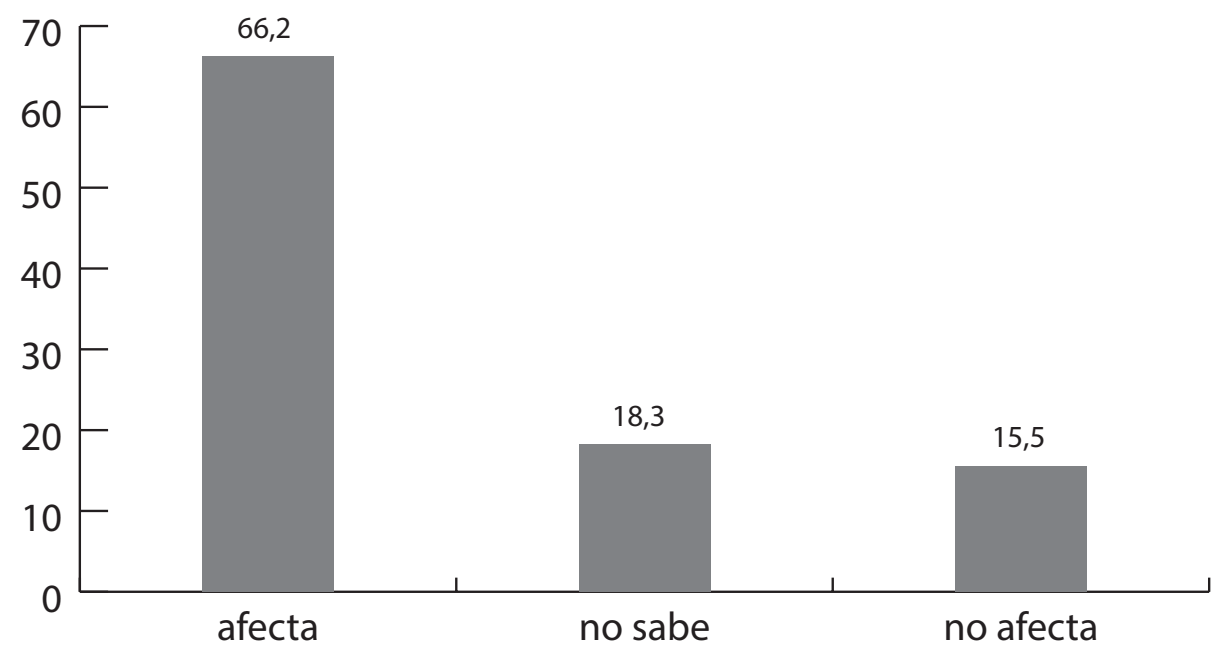

Figura 3. Postura frente a la afección de C. sativa.

La marihuana consumida esporádicamente y en pequeñas dosis promueve distintas alteraciones que van desde lo sensorial, a lo motor entre otras (Monckeberg, 2014). Se encontró que los cannabinoides, impactan negativamente en la memoria a corto plazo, en la memoria de trabajo, en la atención, en la toma de decisiones y la interacción entre los eventos cognitivos y la emoción y quizás tengan plausibilidad con problemas tales como accidentes de tránsito (Torres \& Fiestas 2012). Existiría una predisposición al consumo según la edad, a modo de ejemplo, en los adolescentes está muy extendido y es uno de los factores de riesgo más importantes para el desarrollo de problemas sociales y de salud en edades más avanzadas (Muñoz-Rivas et al., 2005).

Las opiniones marcan que la afección de C. sativa no se correlaciona con los estratos socioeconómicos de las personas, dado que las tres cuartas partes entienden que individuos de todas las condiciones económicas están predispuestos a su afección (Figura 4). 


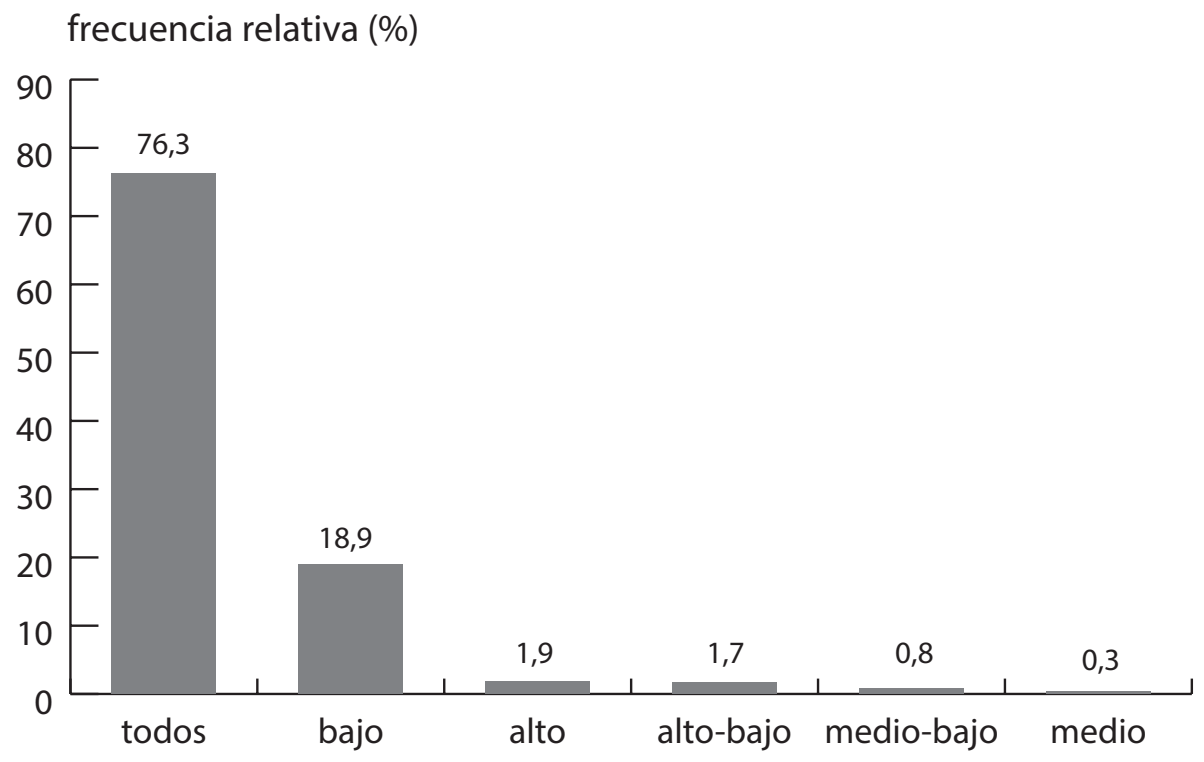

Figura 4. Postura frente a la afección de C. sativa según estrato socioeconómico.

Existen tres variedades de la planta Cannabis: C. sativa, C. indica y C. ruderalis, la primera es de África, el Caribe y América Latina. La variedad índica crece en Asia, mientras que la variedad ruderalis crece en el norte de Europa (Ramos \& Fernández, 2000). En cuanto al conocimiento sobre la biología de la planta, de los respondientes, la gran mayoría identifica la hoja (57\%), asimismo la gran mayoría no tiene idea de los requerimientos de cultivo (Tabla 6). Las semillas de la C. Sativa necesitan condiciones óptimas para desarrollarse, entre las cuales es necesario resaltar factores tales como: temperatura, humedad, fotoperíodo e intensidad luminosa, su germinación es en primavera, las plantas luego comienzan a crecer para florecer en verano, llegando al otoño cuando es posible la cosecha (López et al., 2014). 
Tabla 6

Conocimiento sobre la biología de C. sativa.

\begin{tabular}{|c|c|c|}
\hline Parte identificada & Frecuencia absoluta & Frecuencia relativa $\%$ \\
\hline hoja & 196 & 56,5 \\
\hline flor & 77 & 22,2 \\
\hline aroma & 44 & 12,7 \\
\hline planta & 25 & 7,2 \\
\hline semilla & 5 & 1,4 \\
\hline Total & 347 & 100,0 \\
\hline Cuidados de la planta & Frecuencia absoluta & Frecuencia relativa $\%$ \\
\hline no sabe & 346 & 91,1 \\
\hline luz & 15 & 3,9 \\
\hline calor-luz-agua & 9 & 2,4 \\
\hline calor-luz & 8 & 2,1 \\
\hline luz-agua & 1 & 0,3 \\
\hline calor & 1 & 0,3 \\
\hline Total & 380 & 1,000 \\
\hline
\end{tabular}

Las drogas estimulantes producen: aumento de la atención, estado de alerta, actividad psicomotora y del sistema nervioso autónomo. Actúan sobre mecanismos neurológicos produciendo placer conductual, usurpan los mecanismos dopaminérgicos pero no producen efecto de saciedad, es decir que el aumento de la dopamina no se detiene produciéndose sensibilización dopaminérgica, el mecanismo básico de la adicción (Mazzei, 2018). La planta del Cannabis sp. es químicamente compleja tiene más de 460 componentes que se dividen en cannabidioles y cannabinoles. Los componentes activos del Cannabis sp. son efectivos para el tratamiento del dolor, náuseas (por quimo), anorexia por sida, entre otros, pero se deben evaluar rigurosamente sus efectos (Rodríguez Florez, 2013). Actualmente se ha demostrado que los cannabinoides pueden tener cierta acción terapéutica en: desórdenes neurológicos, lesiones medulares, alteraciones del movimiento, enfermedad de Parkinson, epilepsia y problemas cardiovasculares. (Zozaya Aldana et al., 2011), de hecho una alta proporción de los entrevistados reconoció el efecto medicinal de la marihuana y un vínculo saludable (Tabla 7). 
Tabla 7

Vínculo de los entrevistados con C. sativa y creencia sobre sus usos reales o potenciales.

\begin{tabular}{rcc}
\hline \multicolumn{1}{c}{ Vínculo de la marihuana } & Frecuencia absoluta & Frecuencia relativa \% \\
\hline saludable & 130 & 31,0 \\
narcotráfico & 126 & 30,0 \\
legal & 110 & 26,2 \\
dañina & 54 & 12,9 \\
Total & 420 & 100,0 \\
\hline Creencia sobre los usos & Frecuencia absoluta & Frecuencia relativa \% \\
\hline medicinal recreativo & 188 & 44,76 \\
medicinal & 100 & 23,81 \\
no sabe & 68 & 16,19 \\
recreativo & 60 & 14,29 \\
otros (investigaciones) & 4 & 0,95 \\
Total & 420 & 100,00
\end{tabular}

$\mathrm{El}$ aceite de C. sativa es un producto resinoso y pegajoso que se obtiene al eliminar el solvente de los extractos que contienen los cannabinoides de los cogollos de la planta y que se preparan con diferentes solventes (butano, alcohol isopropílico, etanol o hexano). Su contenido de tetrahidrocannabinol (THC) y cannabidiol CBD puede variar según la variedad del vegetal y las condiciones de elaboración. La técnica de extracción con etanol es una de las más antiguas. En los años cincuenta era conocida como tintura de cannabis y a su vez éste estaba disponible en las farmacias para consumición de cualquier cliente (León Cam, 2017). El THC y sus análogos pueden proporcionar una mejor opción terapéutica en la enfermedad del Alzheimer así como contrarrestar sus síntomas y la progresión de la enfermedad (Eubanks et al., 2006).

Por último una proporción importante de los entrevistados casi $50 \%$ entiende que la liberalización de C. sativa atrae al turismo no obstante lo anterior en Uruguay se exige un registro del consumidor nacional (Figura 5). 


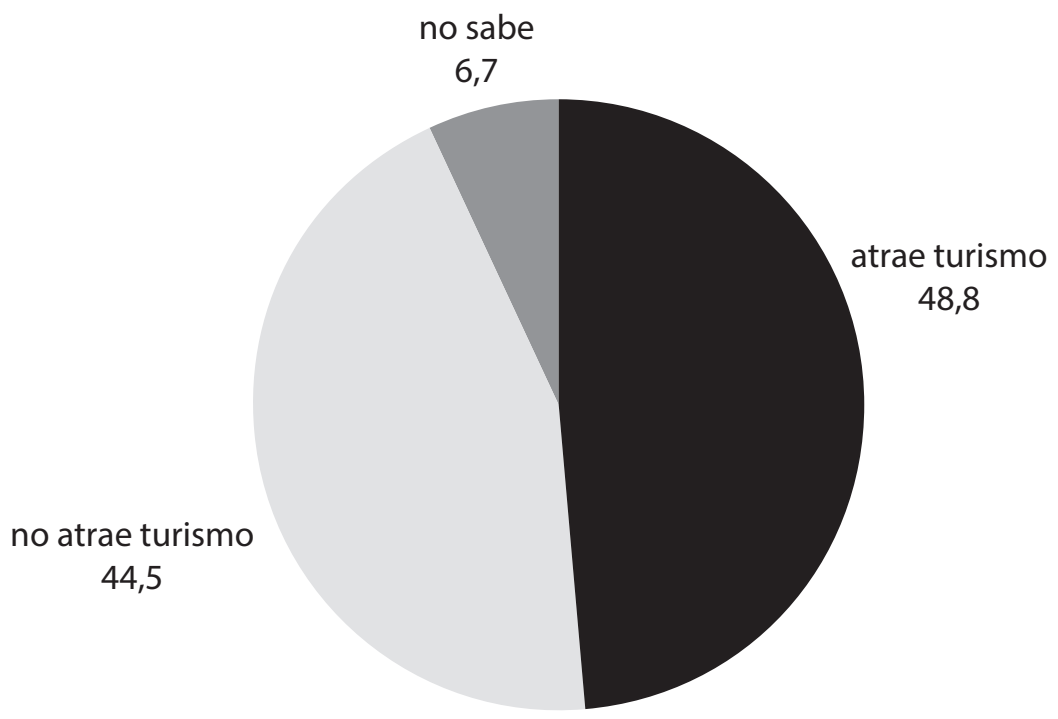

Figura 5. Postura frente a la afección de C. sativa según estrato socioeconómico.

\section{CONSIDERACIONES FINALES}

La línea argumentativa de la legalización del Cannabis pasó por la evidencia de una expansión del consumo correlacionada con un aumento de la legitimidad social, sumado a que las políticas prohibicionistas no habían logrado reducir el consumo; junto a esto la inconsistencia de un consumo legal amarrado a un acceso ilegal que a su vez podía llevar a la adicción de sustancias más fuertes.

Los efectos de C. sativa son muy diversos y duales, es posible establecer que por lo menos un tercio de los uruguayos fronterizos del norte del país, ha probado al menos una vez esta droga por medio de canales principalmente informales, aunque las vías legales producto de la implantación de la ley ya alcanzan un tercio del total, sea por medio de la compra en farmacias o por el autocultivo. Teniendo en cuenta que la gran mayoría de los entrevistados nunca habían consumido cannabis $(68,1 \%)$ es posible entender o correlacionar que también un alto porcentaje de encuestados no conozcan los objetivos de la ley de legalización del cannabis $(77,4 \%)$ y que sólo un $20 \%$ crea que es una forma de combatir el narcotráfico. Es por ello, que la valoración cuantitativa en escala 1-10 sea poco favorable $(4,3)$ y que la mayoría se manifieste en estar en desacuerdo con la ley. 
Otras líneas argumentativas en pro de la legalización del Cannabis pasaron por la generación de un marco legal seguro y propicio para la reducción de daños de otras drogas más tóxicas y adictivas; sumado a ello, el reconocimiento en el terreno de la salud que la marihuana es menos nociva que drogas legales como alcohol y tabaco. No obstante, se encontró en esta investigación que la opinión más general de la población es que este psicoactivo conduce al consumo de otras drogas (tres cuartas partes) y que afecta el trabajo el estudio y la vida social (dos tercios). Además, las tres cuartas partes creen que su consumo es independiente del estrato socioeconómico afectando a todos por igual. En cuanto al reconocimiento de la planta la mayoría la reconoce por la hoja (60\%), sin embargo en relación a su biología el 90\%, desconoce los factores necesarios para el crecimiento y desarrollo de las plántulas. Un 30\% reconoce el poder medicinal de la marihuana y un 50\% entiende que la promulgación de la ley atrae al turismo cannábico.

Es posible que el mercado legal se encuentre desplazando paulatinamente al mercado ilegal provocándole una estrangulación por la falta de dinero que esté dejando de circular; si la hipótesis estuviere ajustada podría asociarse a escenarios de conflictividad en el circuito ilegal con derivaciones ulteriores producto de la reventa de la droga comprada en el circuito legal.

Finalmente, es posible que el impacto de la legalización implique asumir y atender este problema social en las edades tempranas de la educación formal. Durante la formación de ciudadanía quizás será necesario abordar tópicos que marquen el foco en responder a los niños y adolescentes sobre: que es la marihuana, sus efectos de medio y largo plazo, su relación con el consumo de otras drogas, su relación con el embarazo, la libertad de elección sobre las propias conductas, su asociación con conductas de riesgo, su adicción y relación con la habilidad para conducir, su vínculo con las relaciones familiares, el rendimiento académico el trabajo y la vida social y por último la existencia de los posibles tratamientos disponibles en el país. No menos importante resultaría socializar los gastos relacionados con las drogas así como continuar con la medición de impacto de las políticas de control de drogas. Como líneas proyectivas de esta investigación se recomienda el análisis del discurso público y su influencia en la formación de opinión, sobre todo en la prensa, donde las drogas y la marihuana son referidas fundamentalmente como objetos de tráfico y asociadas con la delincuencia. 


\section{REFERENCIAS}

Arocena, F., \& Aguiar, S. (2017). Tres leyes innovadoras en Uruguay: Aborto, matrimonio homosexual y regulación de la marihuana. Revista de Ciencias Sociales, 30(40), 43-62. Recuperado en 21 de junio de 2018, de http://www.scielo. edu.uy/scielo.php?script=sci arttext\&pid=S0797-55382017000100003\&lng=es $\underline{\text { \&tlng=es }}$

Baranger, D. (2006). Construcción y análisis de datos. Introducción al uso de técnicas cuantitativas en la investigación social. In Posadas Canales Cerón, Metodologías de investigación social. $1^{\text {a }}$ ed. Santiago: Lom Ediciones.

CONACE. Comisión Nacional para el Control de Estupefacientes. (2006). Sexto estudio nacional de drogas en población escolar de Chile, 2005, $8^{\circ}$ básico a $4^{\circ}$ medio. Chile: Informe final.

de Armas Bernardi, C. (2016). 'Legalización del cannabis en Uruguay; representaciones sociales y medios de comunicación' Universidad de la República (Uruguay). Facultad de Psicología.

Escobar Toledo, I., Berrouet Mejía, M., \& González Ramírez, D. (2009). Mecanismos moleculares de la adicción a la marihuana. Revista Colombiana de Psiquiatría, 38 (1), 126-142.

Eubanks, L. M., Rogers, C. J., Albert, E., Koob, G. F., Olson, A. J., Dickerson, T. J., \& Janda, K. D. (2006). A molecular link between the active component of marijuana and alzheimer's disease pathology. Mol. Pharm., 3(6) 773-777.

Filardo. V., Aguiar, S., Musto C., \& Pieri, D. (2012). La marihuana provoca esquizofrenia. Espacio público y drogas en Uruguay. Comisión Sectorial de Investigación Científica. UDELAR. Universidad de la República, Uruguay.

Fundación Daya. (2017). Cannabis: Evidencia Científica de uso medicinal, regulación y políticas públicas. Recuperado 22 de agosto de 2018 de http://cannabismedicinal. cl/wp-content/uploads/2017/10/Evidencia-Cannabis-Medicinal-2017.pdf 
Gorosteguy, A., \& Viani, S. (2009). Adolescentes consumidores de marihuana: implicaciones para la familia y la escuela. Revista Salud Mental. Recuperado 29 de agosto de 2018 de http://www.medigraphic.com/cgi-bin/new/resumen. cgi?IDARTICULO $=21989$

INE. Instituto Nacional de Estadística. (2017). Uruguay en cifras. Recuperado de http://www.ine.gub.uy

Infante G. S., \& Zárate de Lara, G. P. (1994). Métodos estadísticos. México: Ed. Trillas.

Instituto Brasilero de Geografía y Estadística. Censo 2010 (2010). Recuperado de https://censo2010.ibge.gov.br

León Cam, J. (2017). El aceite de Cannabis. Revista de la Sociedad Química del Perú, 83 (3), 261-263.

Ley 19. 172-2014. (2014). Legalización de la marihuana. Uruguay. Recuperado de https://www.presidencia.gub.uy/comunicacion/comunicacionnoticias/ promulgacion-regulacion-cannabis

López, A. G., Brindis, F., Cristians Niizawa, S., \& Ventura Martínez, R. (2014). Cannabis sativa L., una planta singular. Revista Mexicana de Ciencias Farmacéuticas, 45 (4). Recuperado de http://www.redalyc.org/articulo.oa?id=57940028004

Marconi, M. A., \& Lakatos, E. M. (2011). Metodologia Científica. 5 ed. São Paulo: Atlas.

Mazzei, E., \& De Souza, M. (2013). La Frontera en Cifras. Melo. Departamento de Cerro Largo 2012. CBA. Montevideo.

Mazzei, J. A. (2018). Problemática del consumo de Cannabis. Revista Americana de Medicina Respiratoria, 18 (2).

Monckeberg, F. (2014). Los pro y contra de la marihuana. Chile. Recuperado de https:// scielo.conicyt.cl/scielo.php?pid $=$ S0370-41062014000200014\&script $=$ sci arttext\&tlng=en

Muñoz-Rivas, M., \& Andreu, J., Gutiérrez P. (2005). Género y continuidad en el consumo de alcohol, tabaco y cannabis en jóvenes. Salud y drogas, 5 (2), 67-79. 
Impacto social de la política de legalización de Cannabis sativa (marihuana) en Uruguay

ONU. Organización de las Naciones Unidas. (2006), Jóvenes y drogas en países sudamericanos: un desafío para las políticas públicas. Primer estudio comparativo sobre uso de drogas en población escolar secundaria.

OTU. (2010). Observatorio territorio Uruguay. Recuperado de http://www.otu.opp. gub.uy/sites/default/files/docsBiblioteca/IDH\%20-\%20S\%C3\%ADntesis\%20 metodol\%C3\%B3gica\%20y\%20de\%20resultados_2.pdf

PNUD. (2010a). Programa Naciones Unidas Desarrollo. Recuperado de http://hdr. undp.org/sites/default/files/hdr 2010 es complete reprint.pdf

PNUD. (2010b). Recuperado de http://www.br.undp.org/content/brazil/pt/home/ idh0/rankings/idhm-municipios-2010.html

Quiroga, P. N., Yohena, I., Contartese, C. M., González, H., \& López, C. M. (2008). Profile of consumption of cocaine, cannabis and opiates in the toxicology laboratory CENATOXA. Acta bioquímica clínica latinoamericana, 42(4), 549555. Recuperado en 29 de agosto de 2018, de http://www.scielo.org.ar/scielo. php?script=sci_arttext\&pid=S0325-29572008000400006\&lng=es\&tlng=en

Ramos, J. A., \& Fernández, J. (2000). Cannabinoides: propiedades químicas y aspectos metabólicos. Adicciones, 12(2), 41-58.

Repetto, L. (2014). Regulación del cannabis: ¿un asunto de seguridad? Entrada y mantenimiento en agenda de un problema de política pública. Revista Uruguaya de Ciencia Política, 23(1), 119-141. Recuperado en 29 de agosto de 2018, de http://www.scielo.edu.uy/scielo.php?script=sci arttext\&pid=S1688-499X20140 $\underline{00100005 \& \operatorname{lng}=\mathrm{es} \& \ln \mathrm{l}=\mathrm{pt}}$

Rodríguez Florez, M. E. (2013). Desafíos que enfrenta el debate y la propuesta de la legalización del Cannabis. TIPS Trabajos de investigación en Políticas Publicas. Recuperado de http://new.econ.uchile.cl/uploads/publicacion/ eb05aa230306d9187a71b345ce8ea11e6f511832.pdf

Rodríguez, R. (2012). Los productos de Cannabis sativa: situación actual y perspectivas en medicina. Salud Mental, Vol. 35, 247-256.

Rodríguez, U., Carrillo, E., \& Soto, E. (2005). Cannabinoides: Neurobiología y usos médicos. Revista Elementos. Vol. 60, 3-9. Recuperado 20 de junio, 2018 de http:// www.elementos.buap.mx/num60/pdf/3.pdf 
Roesch, S. M. A. 2009. Projetos de Estágio e de Pesquisa em Administração. 3a ed. São Paulo: Atlas.

Rovira, F., Decia, J. P., \& La Rosa, G. (2014). La nueva legislación uruguaya sobre marihuana y sus derivados. Cadena Ibero-Americana, (vol 3), 1-12. Recuperado de http://publicaciones.fmdv.org/ojs/index.php/cuadernosderechosanitario/ article/viewFile/130/153

Ruiz. A., \& Prospero. O. (2014). La marihuana. Revista ciencia. Enero-marzo, 62-69. Recuperado de: https://www.revistaciencia.amc.edu.mx/images/revista/65 1/ $\underline{\text { PDF/Marihuana.pdf }}$

Spiegel, M. R., Lipschutz, S., \& Liu, J. 2014. Fórmulas y tablas de matemática aplicada. (4a Ed.) México: McGraw Hill.

Torres, G., \& Fiestas, F. (2012).. Efectos de la Marihuana en la cognición: una revisión desde la perspectiva neurobiológica. Rev Peru Med Exp Salud Publica. 29(1), $127-34$.

UNODC. (2012). Oficina de las Naciones Unidas contra la droga y el delito. Informe Mundial sobre las Drogas. Nueva York: Naciones Unidas. Recuperado de https://www.unodc.org/documents/data-and-analysis/WDR2012/WDR 2012 Spanish web.pdf

Valdés Vento, A. C., Morales Suárez, I. R., Díaz Cabrera, J. C., Sánchez Fuentes, A. L. \& Cuallar Álavarez, R. (2009). Criterios de evaluación de la variable "pertinencia e impacto social" específicos para la carrera de Medicina. Educación Médica Superior, 23(3), 45-62. Recuperado de http://scielo.sld.cu/pdf/ems/v23n3/ ems05309.pdf

Zozaya Aldana, B., Medina Rodriguez, I., \& Tamayo Pineda, N. (2011). Cannabinoides y su posible uso en el glaucoma. Revista Cubana de Farmacia, 45(3), 439448 Recuperado en 30 de agosto de 2018, de http://scielo.sld.cu/scielo. php?script=sci arttext\&pid=S0034-75152011000300013\&lng=es\&tlng=es 